\title{
Design Considerations for High-Frequency Coaxial Winding Power Transformers
}

\author{
Mark S. Rauls, Donald W. Novotny, Fellow, IEEE, and Deepakraj M. Divan, Senior Member, IEEE
}

\begin{abstract}
The use of coaxial windings to create low loss, low leakage reactance, power transformers for use in high frequency soft switched $\mathrm{dc} / \mathrm{dc}$ and resonant converters has been demonstrated in [1]. This paper examines some of the important loss aspects of the design of coaxial winding transformers such as the influence of skin effect on winding resistance, the variation of core loss caused by nonuniform core flux density, and the choice of the principle dimensions and aspect ratios for maximum efficiency. Experimental measurements on a $50 \mathrm{kVA} 50 \mathrm{kHz}$ unit, are included to confirm portions of the analytical results and suggested design procedures.
\end{abstract}

\section{INTRODUCTION}

O NE OF THE MORE important concerns in high frequency power conversion is the question of magnetic component design, particularly for higher power levels. The question of how to realize high-power high-frequency transformers has been particularly daunting. It is extremely important to obtain very low leakage inductance values, while simultaneously ensuring that the leakage flux is not concentrated in a small section of the core used. Further, transformer designs which feature an easily calculated leakage inductance are appealing for use in soft switching circuits in which the leakage inductance is a useful circuit component.

The use of coaxial transformers for high-frequency, highpower converters was proposed in [1]. The coaxial transformer concept has been used with considerable success in various converters including a $50 \mathrm{kHz}, 50 \mathrm{~kW}$ dual active bridge dc/dc converter and a 600 watt, $1 \mathrm{MHz}$ dual resonant dc/dc converter $[2,3]$. The design of such coaxial transformers is considerably different from that of conventional transformer structures and needs to be better understood. This paper investigates the losses associated with the coaxial transformer configuration shown in Fig. 1.

The transformer consists of an outer conducting tube which forms one of the windings. As can be seen, the inner winding is wound completely inside the outer conductor. Integral turns ratios are possible by using multiple turns on the inner winding. The magnetic core is outside the outer conductor. Since

Paper IPCSD 92-95, approved by the Industrial Power Converter Committee of the IEEE Industry Applications Society for presentation at the 1991 IAS Annual Meeting, Dearborn, MI, September 28-October 4. This work was supported by the Wisconsin Electric Machines and Power Electronic Consortium (WEMPEC).

M. S. Rauls was with the Department of Electrical and Computer Engineering, University of Wisconsin-Madison, Madison, WI 53706. He is now with EMD Associates, Winona, MN 55987.

D. W. Novotny and D. M. Divan are with the Department of Electrical and Computer Engineering, University of Wisconsin-Madison, Madison, WI 53706.

IEEE Log Number 9207098.

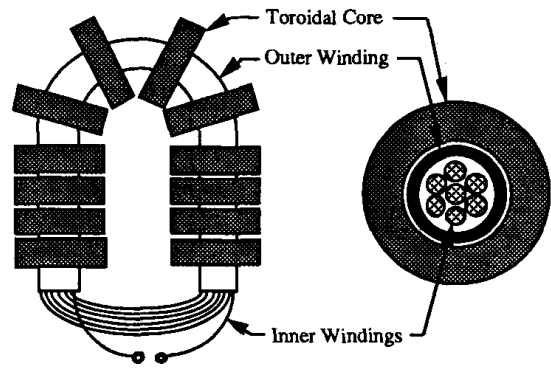

Fig. 1. Coaxial transformer arrangement.

the magnetic configuration of a coaxial winding transformer is significantly different from conventional transformers, a number of unique design issues exist. Some of these are explained in the following sections.

\section{FluX Distribution AND CORE LosS}

When toroidal cores are used, the core flux distribution is well defined and controlled. Essentially none of the leakage flux finds its way into the core. The core flux distribution is, however, nonuniformly spread across the radius of the core, and indicates that core loss depends on the ratio of the toroidal core inner $\left(r_{c i}\right)$ and outer $\left(r_{c o}\right)$ radii.

Ferrite manufactures often specify core loss as a log-log plot of total power loss per unit volume versus flux density at specific frequencies. In general, loss data is obtained using toroids whose ratio of $r_{c o}$ to $r_{c i}$ is sufficiently close to unity so that the flux density is approximately uniform in the core under test.

To extend the core loss data to "fat" toroids where the flux density cannot be assumed uniform a closed form expression is curve fitted to the manufacture's core loss plot for the material and frequency of interest. In general, the core loss per unit volume $\left(C_{l v}\right)$ is of the form

$$
C_{l v}=K_{c}(B)^{n}
$$

where $B$ is the spatial varying flux density, $n$ is an exponent usually near 2.5 , and $K_{c}$ is the core loss units/scaling constant. The actual flux density in the core will vary as

$$
B=\frac{K_{B}}{r}
$$

where $K_{B}$ is the flux density units/scaling constant, and $r$ is the radial distance from the coaxial center. Substitution of (2) 


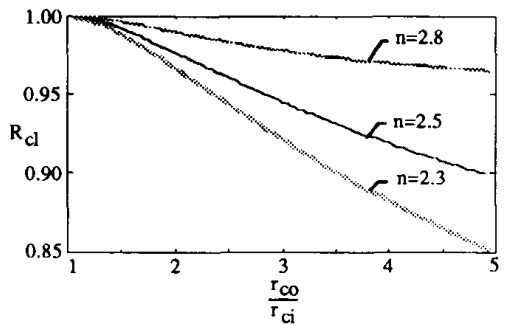

Fig. 2. Core loss ratio $\left(R_{c l}\right)$ for $\left(1<\left(r_{c o} / r_{c i}\right)<5\right)$.

into (1) gives

$$
C_{l v}=K_{c}\left(\frac{K_{B}}{r}\right)^{n}
$$

which is the core loss per unit volume as a function of position in the core. Integration of (3) over the core volume gives the total core loss. The total core loss resulting from nonuniform flux distribution is compared to a calculation using uniform flux distribution to show the effect on total core loss. The constraint for comparison is that the total flux and core volume be the same for both calculations.

The ratio $\left(R_{c l}\right)$ of total core loss for nonuniform flux distribution to the total core loss for uniform flux distribution is

$$
R_{c l}=\frac{2\left(1-\frac{r_{c i}}{r_{c o}}\right)^{n-1}\left(1-\left(\frac{r_{c i}}{r_{c o}}\right)^{2-n}\right)}{(2-n)\left(\ln \left(\frac{r_{c o}}{r_{c i}}\right)\right)^{n}\left(1+\frac{r_{c i}}{r_{c o}}\right)}
$$

for $n \neq 2$. When $n=2$, (4) involves an additional logarithm, but the effect is the same. Notice that $R_{c l}$ only depends on the core radii and the material dependent exponent $n$. For "thin" toroids where the ratio of $r_{c o}$ to $r_{c i}$ is close to one the total core loss ratio of (4) will also be close to one. However, the ratio is actually less than one as the toroid becomes more "fat." This result indicates that core losses are actually lower when nonuniform flux distribution is taken into account. The effect is small for toroids of practical dimensions and indicates that using the "approximation" of uniform flux density for core loss calculations is a good one. This is contrary to intuition. Qualitatively, the actual flux density is minimum at $r_{c o}$ and maximum at $r_{c i}$ which results in the majority of core volume being less lossy, and a small portion of volume being more lossy than the uniform approximation predicts. The core loss ratio (4) is plotted in Fig. 2 for several values of $n$ and can be used to improve the uniform core loss approximation.

The above discussion and (4) were derived by ignoring core saturation (i.e., constant permeability was assumed). With core saturation included, the plots of Fig. 2 would become more flat because saturation has the effect of evening out any nonuniform flux distribution. Hence, with saturation included in the analysis, the actual core loss will look even more like the core loss approximation which assumes uniform flux.

\section{SKIN EFFECT AND WINDING LOSSES}

The windings of a coaxial transformer are essentially a coaxial cable and therefore much of the basic theory associated

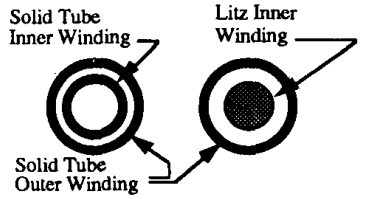

Fig. 3. Examples of $1: 1$ cylindrical symmetry windings.

with coaxial lines can be applied. However, unlike most applications for coaxial lines, these transformers are required to operate at high current levels. One of the concerns this raises is the current distribution in the inner and particularly the outer conductor and the effect on winding losses.

\section{A. Losses in the Outer Winding with Sinusoidal Excitation}

The current distribution in the outer tube is identical to a coaxial transmission line when the transformer windings have one turn cylindrical symmetry and the net current in the outer and inner windings is equal and opposite. Two examples of this symmetry are shown in Fig. 3. In a real coaxial transformer the currents will not be exactly equal because whichever winding is used as the primary will also carry the magnetizing current. However, this effect is small and can be neglected because the coaxial transformer requires very little magnetizing current (e.g., typically less than $1 \%$ full load). When multiple turns of litz wire are used for the inner winding there is no longer cylindrical current distribution in the inner winding and there will be some proximity effect at the outer tube. However, if the windings are centered, as in Fig. 1, the effect on the outer tube current distribution should be small, and the coaxial transmission line approximation should give good results. Future work will examine the validity of this approximation.

Assuming cylindrical symmetry as in Fig. 3, and sinusoidal current flow, the current distribution in the outer winding is described by Bessel's differential equation of order zero

$$
\frac{d^{2} \boldsymbol{J}}{d r^{2}}+\frac{l d \boldsymbol{J}}{r d r}-j \frac{\omega \mu_{0}}{\rho} \boldsymbol{J}=0
$$

where $\boldsymbol{J}$ is the current density (magnitude and phase), and $r$ is the radial distance from the axis of symmetry. The general solution of (5) involves a linear combination of the modified Bessel functions $I_{0}$ and $K_{0}$ and is worked out in many textbooks [4], [5]. Boundary conditions on the outer tube are that there is no magnetic field at the outer surface $\left(r_{t o}\right)$, because the net current enclosed is zero, and that the magnetic field resulting from the current in the inner winding at the inner surface $\left(r_{t i}\right)$ is tangential. Using the solution to (5) and an approach similar to one presented in [5], the ac resistance of the outer winding per meter $\left(R_{a c_{-o}}\right)$ is found to be

$$
R_{a c_{-o}}=\operatorname{Real}\left(\begin{array}{c}
f \mu_{0} \delta e^{j \frac{\pi}{4}\left(I_{0}\left(b_{o}\right) K_{1}\left(a_{o}\right)\right.} \\
\frac{\left.+I_{1}\left(a_{o}\right) K_{0}\left(b_{o}\right)\right)}{\sqrt{2} b_{o}\left(I_{1}\left(a_{o}\right) K_{1}\left(b_{o}\right)\right.} \\
\left.-I_{1}\left(b_{o}\right) K_{1}\left(a_{o}\right)\right)
\end{array}\right)[\Omega / m]
$$

where

$$
a_{o}=\frac{\sqrt{2} r_{t o}}{\delta} e^{j \pi / 4}
$$




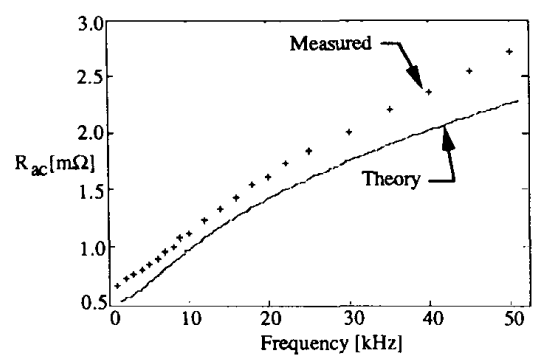

Fig. 4. Measured and theoretical resistance of coaxial pipe.

and

$$
b_{o}=\frac{\sqrt{2} r_{t i}}{\delta} e^{j \pi / 4}
$$

If the inner winding is also a solid hollow cylinder, the ac resistance of the inner winding can be found using the same method with different boundary conditions. Boundary conditions for the inner tube are that there is no magnetic field at the inner surface because there is no enclosed current, and that the magnetic field at the outer surface of the inner tube is tangential. The resulting resistance per meter $\left(R_{a c_{-i}}\right)$ is

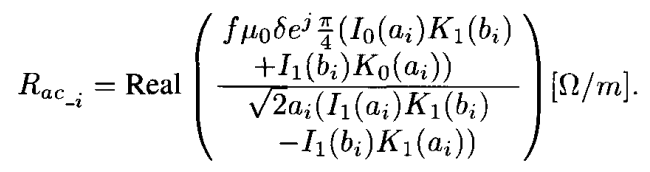

The theoretical results of (6) and (9) were compared to resistance measurements of a coaxial conductor in the lab under sinusoidal excitation. Two 1.5 meter solid copper pipes were placed concentric to one another similar to Fig. 3. At one end the pipes were soldered together and at the other end the pipes were driven by a current source maintained at $90 \mathrm{~A}_{r m s}$. In this configuration the pipes are essentially two resistors in series, but carrying equal and opposite current. The highest frequency used was $50 \mathrm{kHz}$ so that transmission line effects would not be present. The effective ac resistance of the coaxial pipe was found by measuring the input power and current in the pipes on the LeCroy digital oscilloscope and then solving for the resistance using

$$
R_{a c}=\frac{P_{\text {ave }}}{I_{\mathrm{rms}}^{2}} \Omega
$$

The theoretical and measured resistances of the coaxial pipe versus frequency are plotted in Fig. 4. The measured values agree well with the theory, but are slightly higher than the theory predicts which is believed to be caused by contact and solder joint resistances.

A useful design curve can be obtained by plotting (6) normalized to the dc equivalent resistance at one skin depth $(\delta)$, versus tube thickness normalized to one $\delta$. The curve is plotted in Fig. 5 for several ratios of the outer tube's inner radius normalized to skin depth $\left(R_{t i} / \delta\right)$.

The results of Fig. 5 indicate that there is actually an optimum wall thickness for the outer tube in order to minimize the resistance of the outer tube at a particular frequency. The curve with $R_{t i} / \delta=0.2$ does not represent tube dimensions of

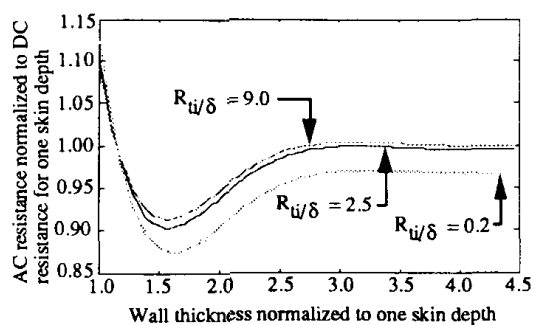

Fig. 5. ac resistance of outer winding versus tube thickness.

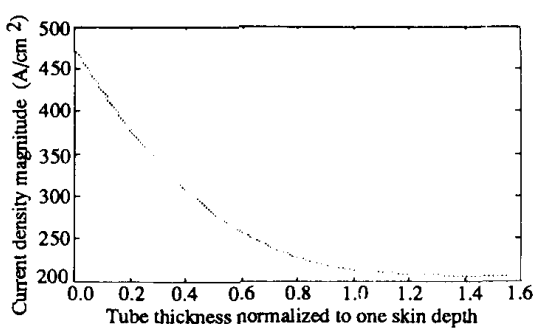

Fig. 6. Distribution of current density magnitude, outer tube.

practical size, but shows that the curve is somewhat dependent on the inner radius to skin depth ratio. With $R_{t i} / \delta$ in the range $\left(4<R_{t i} / \delta<\infty\right.$ ) the curve lies essentially where the $R_{t i} / \delta=9.0$ curve is in the figure and represents practical tube dimensions and frequencies of interest. As the tube thickness approaches $4 \delta$ the ac resistance is equivalent to the dc resistance of a tube one skin depth thick with the constraint that the inner radius is the same. The minimum resistance occurs when the wall thickness is $1.55 \delta$. The resistance for a tube which has a wall thickness significantly less than $\delta$ is approximately the $\mathrm{dc}$ resistance of the tube.

It is useful to look at the current density distribution to understand the minimum resistance phenomenon of Fig. 5. The current density magnitude is plotted in Fig. 6 for an outer winding tube thickness of $1.55 \delta$ carrying a net current of $200 \mathrm{~A}_{r m s}$. The current density magnitude exhibits the exponential shape and crowding of the current towards the inner surface of the outer conductor which is expected for a coaxial transmission line. The current density phase is plotted in Fig. 7. The phase difference between the current at the two surfaces of the tube is $90^{\circ}$. Tubes with thicker walls would experience a phase shift greater than $90^{\circ}$ which would result in some current flowing in the opposite direction of the net current flow, which results in increased resistance even though the tube is thicker.

\section{B. Losses in the Outer Winding with Nonsinusoidal Current}

The above analysis examined the ac resistance of the outer tube for sinusoidal excitation which is not all that useful by itself for power electronic applications. The usual situation involves a transformer driven by a square wave voltage, with a triangular current waveform resulting from inductive switching. In terms of outer winding losses, a Fourier series representation can be used on the current waveform in conjunction with the ac resistance at each harmonic to 


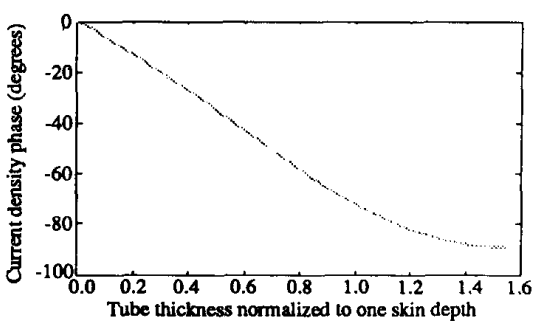

Fig. 7. Variation of current phase in outer tube.

determine the total loss. If the designer uses a tube thickness of $1.55 \delta$ to minimize the ac resistance at the fundamental, the remaining harmonic currents will essentially see the dc equivalent resistance for one skin depth at each harmonic. The resulting ac resistance of the outer tube with a triangular current waveform is $25 \%$ greater than when the current waveform is sinusoidal for the same rms current.

\section{Losses in the Inner Winding(s)}

Single Turn Solid Cylindrical Winding: The ac resistance for this type of winding was introduced above and is described by (9). A solid tube inner winding exhibits the same minimum resistance phenomenon as the outer tube winding. By constructing a curve similar to the one in Fig. 5, one can show that the minimum resistance occurs at a wall thickness of $1.55 \delta$. This is the same optimum thickness as the outer tube winding and the shape of the curve is identical to Fig. 5.

Single Turn Litz Winding: Litz wire is typically used over solid or stranded wire when high frequency and high current capacity is needed in transformers and inductors. The ac resistance of litz wire can be found using the manufacture's data sheets and is typically close to the $\mathrm{dc}$ equivalent of the individual strands in parallel when the gauge on the individual strands is appropriate for the frequency of interest. If the litz wire is centered in the outer tube there will be no difference in the current distribution and losses compared to the case of an isolated litz wire carrying an equal amount of current.

Multiturn Litz Winding: When there are multiturns used as the inner winding there is no longer coaxial symmetry between the inner and outer winding and the proximity effect needs to be looked at for precise loss calculations. However, proximity effect is minimized by the litz wire, and good results can be obtained by ignoring the fact that the windings are not centered (i.e., assume uniform current distribution in the litz conductor and compute the ac resistance as if there were a single turn present).

\section{OPTIMUM EFFICIENCY DESIGNS}

Transformer designs are essentially governed by the amount of core needed to avoid saturation and the amount of winding cross sectional area needed to support the full load current. It has been shown [1] that one of the main advantages to the coaxial transformer design is its low leakage reactance which is also precisely controllable and hence a useful circuit component. The leakage reactance is primarily confined to the inner winding side of the transformer as shown in the

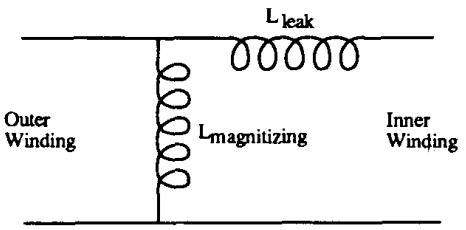

Fig. 8. Equivalent circuit showing asymmetrical leakage.

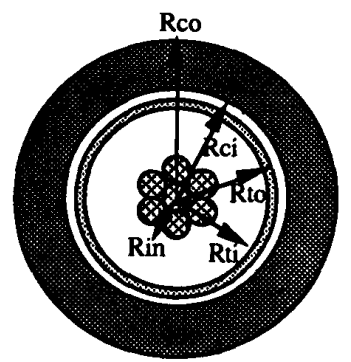

Fig. 9. Radii for the coaxial transformer.

asymmetrical circuit of Fig. 8 [3]. This essentially adds an additional constraint to the design if one intends to use the leakage reactance as an actual circuit component rather than simply trying to minimize it.

\section{A. Design Procedure}

As an initial design, the desired current capacity and choice of conductor type with appropriate fill factor determines the equivalent outer radius $\left(r_{i n}\right)$ of the inner winding as shown in Fig. 9. The leakage inductance on the inner winding side was shown to be

$$
L_{\text {leak }}=\frac{N_{w}^{2} \mu_{o}}{8 \pi}\left(1+4 \ln \left(\frac{r_{t i}}{r_{i n}}\right)\right)[H / m]
$$

in [1], where $r_{t i}$ is the inner radius of the primary tube and $N_{w}$ is the number of inner winding turns. This relation is exact for a $1: 1$ turns ratio, but is an approximation when the inner winding has multiple turns or when there is significant skin effect. The exact leakage reactance under these conditions needs to be addressed in future work. By knowing the desired leakage reactance and selecting a transformer length, one can determine $r_{t i}$ from (11). The results in the previous section on winding losses indicate that the outer tube resistance will be minimized when the wall thickness is $1.55 \delta$. However, if the outer winding is to provide structural integrity to the transformer, the outer tube wall thickness may need to be several $\delta$ at multi-kHz frequencies. With the outer tube wall thickness determined, the inner radius of the toroidal cores can be determined after allowing necessary space for insulation between the core and outer tube winding.

If the leakage reactance is not a desired component and is to be minimized, the space between the outer tube and the inner winding(s) should be minimized. Under this condition the crowding of the current towards the inner surface of the outer conductor could become a problem in the presence of 


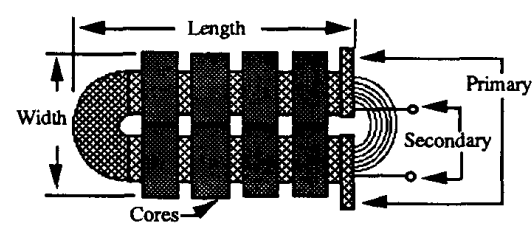

Fig. 10. Transformer length and width definitions.

significant skin effect since the outer tube will have significantly less cross-sectional area compared to an assumed litz wire for the inner winding. This problem could be solved by using coaxial litz cable so that both the outer and inner windings are formed of litz wire.

The remaining constraint of the transformer design is to determine the necessary flux cross-sectional area for the transformer core. The core cross sectional area $\left(A_{c}\right)$ is determined by the transformer voltage $\left(V_{r m s}\right)$, maximum flux density for the core material being used $\left(B_{m}\right)$, frequency of interest $(f)$, and the number of turns $(N)$. This relation is derived in many textbooks [6] and is repeated here:

$$
V_{\mathrm{rms}}=K_{f} N f B_{m} A_{c}
$$

where the form factor $\left(K_{f}\right)$ is 4.0 for square wave excitation or 4.44 for a sinusoidal waveform.

An efficiency optimization can be done on the initial dimension choices by iterating on the transformer length until losses are minimized. Keeping the inner winding cross-sectional window fixed and using constraints (11) and (12), one can compute the losses as a function of length to width ratio. See Fig. 10 for clarification of the transformer length and width definitions. Core loss will be minimized for geometries which maximize cross sectional area per unit volume. This implies using a core which has a thin profile and a small inner radius. Winding losses will be minimized when the winding length is minimized. The total minimum loss will occur for a geometry somewhere in the middle of these extremes.

\section{B. Efficiency Optimization for a $50 \mathrm{kVA}, 50 \mathrm{kHz}$ Unit}

Theoretical losses are examined in the following optimization example for a $50 \mathrm{kVA}, 50 \mathrm{kHz}$ transformer which was built for experimental work done in [1] and [2]. The desired leakage reactance and current rating was used to select the winding geometry in the original design. The core geometry chosen was limited by practical considerations such as readily available ferrite cores with an inner core radius appropriate for the size of the outer tube winding. In the optimization process the inner winding cross sectional area remains fixed and the outer winding is allowed to vary so that as the length changes, the leakage reactance remains fixed. As the transformer length is varied the outer tube radius is determined using (11). This determines the core inner radius, and the core outer radius is adjusted so that the flux cross-sectional area remains fixed. The losses are calculated at each iteration of the dimensions using the core and winding loss results of the previous sections. Fig. 11 shows the resulting variation in losses as a function of the transformer length to width ratio as defined in Fig. 10.

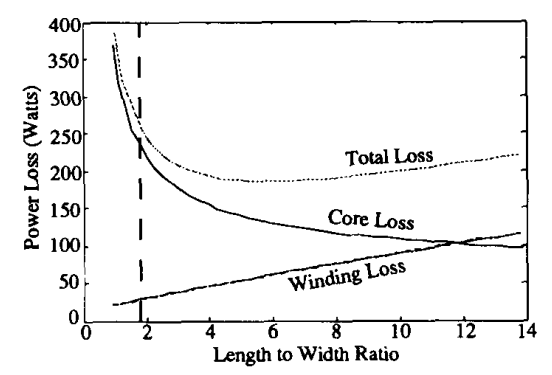

Fig. 11. Winding, core, total loss versus length-to-width ratio.

The results of Fig. 11 indicate that a length-to-width ratio near 6 provides the minimum loss using the initial design constraints. The optimum transformer design is long and thin because the core loss dominates in this example, and core loss will be minimum with long and thin cores. The length-to-width ratio of the transformer built in the lab is 1.8 . The dashed line in Fig. 11 shows where the experimental transformer lies in comparison to the optimal point. The curve shows that the transformer loss could be reduced by $30 \%$ if the optimal loss dimensions were used over the initial design dimensions.

\section{Optimum Efficiency Length-to-Width Ratios}

In the above optimization example it was shown that the transformer profile for maximum efficiency was rather long and thin. This is atypical since maximum efficiency designs for conventional electric machines and transformers tend to be cubical in shape. Without some means of forced cooling, coaxial transformers will tend to have long-thin profiles so that the transformer temperature rise is kept under control. The windings are almost entirely surrounded by core which makes it difficult to dissipate heat. When the current density is constrained to maintain acceptable temperature rise, the loss per unit volume for the windings will tend to be lower than the loss per unit volume of the core. Hence, for maximum efficiency designs, the transformer profile will favor minimum core volume which implies long-thin toroidal cores to provide the necessary flux cross sectional area at the minimum volume.

Heat removal from the transformer occurs primarily from the surface, which is core material irrespective of the transformer profile. Consequently, a long-thin profile for the transformer is advantageous as surface area is maximized. However, it should be noted that as the core is typically a poor thermal conductor, short transformer designs may benefit from additional heat removal along the primary/secondary conductors to the outside and to the end windings.

Transformer performance can be substantially improved if forced convection heat transfer is implemented. Forcing a coolant through the primary tube would allow a dramatic increase in the current handling capability of the transformer, and thus impact the power rating. This is not as easily accomplished in conventional transformers because voltage drop across the leakage inductance at the higher current levels can become a significant limitation. 
TABLE I

Measured Theoretical $50 \mathrm{kVA}, 50 \mathrm{kHz}$ Transformer loss

\begin{tabular}{llll}
\hline & Theory & Measured & $\%$ diff. \\
Core Loss & $240 \mathrm{~W}$ & $231 \mathrm{~W}$ & $4 \%$ \\
Winding Loss & $33 \mathrm{~W}$ & $69 \mathrm{~W}$ & $109 \%$ \\
Efficiency & $99.5 \%$ & $99.4 \%$ & $0.1 \%$ \\
\hline
\end{tabular}

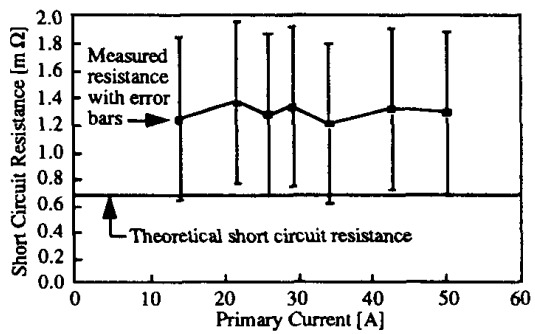

Fig. 12. Measured and theoretical winding resistance.

\section{Measured Versus Theoretical Losses, $50 \mathrm{kVA}, 50 \mathrm{kHz}$ Unit}

Measured losses for the $50 \mathrm{kVA}, 50 \mathrm{kHz}$ transformer are compared to the theoretical predictions. The measurements are taken under sinusoidal voltage and current excitation. Table I summarizes the loss results.

Winding Losses: Short circuit measurements were taken using 10 gauge litz wire soldered together at the end to form a 7 turn short circuited inner winding secondary. The outer tube served as the transformer primary. The losses could not be measured at full load current due to equipment limitations. The full load winding losses were determined by measuring the input power and current into the transformer on the LeCroy digital oscilloscope and then solving for the resistance using (10). The measurement has an error bound which is determined from the phase and magnitude accuracy of the current probe, digitizing resolution of the oscilloscope, and the accuracy of reading a current value from the scope display. The measured resistance with error bars showing the error bound is compared to the theoretical resistance of the transformer in Fig. 12. The theoretical resistance for the outer and inner winding is found using the winding resistance results of the previous section. In order to compare the theoretical and measured results, the inner winding resistance is referred to the outer winding side and added to the outer winding resistance so that the resistance shown in Fig. 12 is the total resistance looking into the transformer.

The results of Table I and Fig. 12 show that there is a large discrepancy between the theoretical value and the measured transformer resistance. It is difficult to precisely measure the resistance because the transformer is almost entirely inductive. The error bound on the measurement is $0.6 \mathrm{~m} \Omega$ which is almost equal to the theoretical resistance. Also, the theoretical losses do not account for the solder joint resistance in the short circuited litz wire and the outer winding connections which could have resistances comparable to the resistance of the windings by themselves.
Core Loss: The core loss for the transformer is measured with the secondary open circuited. The outer tube winding is used as the primary. Essentially all of the applied voltage is across the transformer magnetizing reactance due to the extremely small leakage reactance and resistance of the outer winding. Hence, the core loss is the measured input power to the transformer at rated voltage. The theoretical core loss is found using the manufacture's core loss data and the core loss results presented earlier. Table I shows that the measured and theoretical core loss agree very well.

The core loss was also measured using square wave voltage excitation with the amplitude set so that the peak flux density was the same as in the sinusoidal case. The measured results indicate that the core losses are essentially the same for both waveforms as long as the flux density is the same. This is because ferrite core loss is mostly hysteretic and only depends on the peak flux density when driven at flux densities near the saturation flux density.

\section{CONCLUSIONS}

The loss aspects of the coaxial winding transformer have been investigated. It has been shown that nonuniform flux distribution in the toroidal core has little effect on the core loss, and that computing the core loss by assuming uniform flux density in the core gives a conservative and good estimate of the core loss. Minimum ac resistance at the fundamental frequency component in the outer tube winding is achieved by making the wall thickness 1.55 skin depths thick. If the inner winding is also a hollow cylinder its resistance is also minimized by using a wall thickness of 1.55 skin depths.

A design methodology for achieving optimum transformer efficiency has been presented. Optimum efficiency designs for coaxial transformers tend to have nonconventional aspect ratios which are long and thin. This long-thin profile is also advantageous for heat transfer due to the increased surface area. More conventional aspect ratios will result from improved cooling techniques which allow higher current densities and therefore a more even balance of the transformer losses between the windings and the core.

\section{REFERENCES}

[1] M. H. Kheraluwala, D. W. Novotny, and D. M. Divan, "Design considerations for high frequency transformers," in IEEE-PESC' 90 Conf. Rec., pp. 734-742.

[2] M. H. Kheraluwala, R. W. Gascoigne, D. M. Divan, and E. Bauman, "Performance characterization of a high power dual active bridge DC/DC converter", in IEEE-IAS'90 Conf. Rec., pp. 1267-1273.

[3] H. L. N. Wiegman, D. M. Divan, D. W. Novotny, and R. Mohan, "A ZVS dual resonant converter for battery charging applications," to be published in IEEE-PESC'91 Conf. Rec.

[4] W. C. Johnson, Transmission Lines and Networks. New York: McGrawHill, 1950.

[5] W. R. Smythe, Static and Dynamic Electricity, 3rd ed. New York: McGraw-Hill, 1968

[6] A. Goldman, Modern Ferrite Technology. New York: Van Nostrand Reinhold, 1990. 


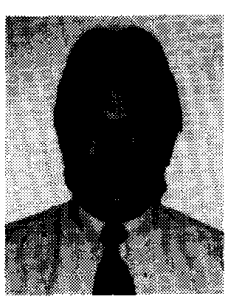

Mark S. Rauls received the A.A.S. degree in electronic technology from Madison Area Technical College in 1980 and the B.S. and M.S. degrees in electrical engineering from the University of Wisconsin, Madison, in 1988 and 1992, respectively.

From 1980 to 1983, he was employed by AT\& Bell Laboratories as a software developer for telephone switching systems. From 1988 to 1990 , he was employed by Honeywell as a development engineer. While there, he was involved with the development of gas furnace ignition controls for residential furnaces. He is presently employed at EMD Associates, Inc. as a design engineer.

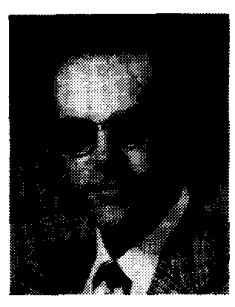

Donald W. Novotny (M'62-SM'77-F'87) received the B.S. and M.S. degrees in electrical engineering from the Illinois Institute of Technology, Chicago, in 1956 and 1957, respectively, and the $\mathrm{Ph} . \mathrm{D}$. degree from the University of Wisconsin, Madison, in 1961.

Since 1961, he has been a member of the faculty at the University of Wisconsin, Madison, where he is currently Professor and Co-Director of the Wisconsin Electric Machines and Power Electronics Consortium (WEMPEC). He served as Chairman of the Electrical and Computer Engineering Department from 1976 to 1980 and as an Associate Director of the University-Industry Research Program from 1972 to 1974 and from 1980 to the present. He has been active as a consultant to many organizations and a Visiting Professor at Montana State University, Boseman, the Technical University of Eindhoven, Eindhoven, The Netherlands, the Catholic University of Leuven, Leuven, Belgium, and a Fullbright Lecturer at the University of Ghent, Ghent, Belgium. His teaching and research interests include electric machines, variable-frequency drive systems, and power electronic control of industrial systems.

Dr. Novotny is a member of ASEE, Sigma Xi, Eta Kappa Nu, and Tau Beta Pi and is a Registered Professional Engineer in the State of Wisconsin.

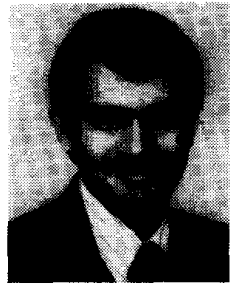

Deepakraj M. Divan (M'83-SM'92) received the B.Tech. degree in electrical engineering from the Indian Institute of Technology, Kanpur, India, and the M.Sc. and Ph.D. degrees in electrical engineering from the University of Calgary, Calgary, Canada, in 1975,1979 , and 1983 , respectively.

He worked for two years as a Development Engineer with Philips India Ltd. In 1979, he started his own concern in Pune, India, providing product development and manufacturing services in the power electronics and instrumentation areas. In 1983, he joined the Department of Electrical Engineering at the University of Alberta as an Assistant Professor. Since 1985, he has been with the Department of Electrical and Computer Engineering at the University of Wisconsin, Madison where he is presently a Professor. He is also an Associate Director of the Wisconsin Electric Machines and Power Electronics Consortium (WEMPEC). His primary areas of interest are in power electronic converter circuits and control techniques. He has written more than 25 papers in the area and has been granted many patents.

Dr. Divan was a recipient of the Killam Scholarship and a co-recipient of the 1988 Third Prize Paper Award from the Power Semiconductor Committee and the 1983 Third Prize Paper Award from the Static Power Converter Committee of the IEEE Industry Applications Society. He is also a consultant for various industrial concerns. He was the Program Chairman for the 1988 and 1989 Static Power Converter Committee of the IEEE-IAS, Program Chairman for PESC 91, and a Treasurer for PESC 89. He is also a Chairman of the Education Committee in the IEEE Power Electronics Society. 